\title{
DISPERSION OF A NEW CORONAVIRUS SARS-COV-2 BY AIRLINES IN 2020: TEMPORAL ESTIMATES OF THE OUTBREAK IN MEXICO
}

\author{
Gustavo Cruz-Pacheco ${ }^{1}$, José F. Bustamante-Castañeda ${ }^{2}$, Jean G. Caputo ${ }^{3}$, \\ María E. Jiménez-Corona ${ }^{4,5}$, ANd Samuel Ponce-De-León-Rosales ${ }^{6}$
}

${ }^{1}$ Department of Mathematics and Mechanics, Instituto de Investigaciones en Matemáticas Aplicadas y en Sistemas (IIMAS), National University of Mexico (UNAM), Mexico City; ${ }^{2}$ Graduate Program in Mathematical Sciences, UNAM, Mexico City ; ${ }^{3}$ Laboratory of Mathematics, Institut National des Sciences Appliquées de Rouen Normandie, SaintEtienne du Rouvray, France; ${ }^{4}$ Department of Epidemiology, Instituto Nacional de Cardiología Ignacio Chávez, Mexico City; ${ }^{5}$ Division of Graduate Studies and Research, Facultad de Odontología, UNAM, Mexico City; ${ }^{6}$ Programa Universitario de Investigación en Salud, UNAM, Mexico City, Mexico

\begin{abstract}
Background: On January 23, 2020, China imposed a quarantine on the city of Wuhan to contain the SARS-CoV-2 outbreak. Regardless of this measure, the new infection has spread to several countries around the world. Objective: We developed a method to study the dissemination of this infection by airline routes and provide estimations of the time of arrival of the outbreak to different cities. Methods: Using the Kermack and McKendrick model complemented with diffusion on a graph composed of nodes and edges, we made an analysis of COVID-19 dispersion to other cities by air travel. Results: The estimation was accurate in that it was possible to predict in the middle of February 2020 the arrival of the first outbreak in Mexico, which eventually occurred between March 20 and 30. This estimation was robust with respect to small changes in epidemiological parameters at the other nodes. Conclusions: The estimation of the time of arrival of the outbreak from its epicenter, allows for a time period to implement and strengthen preventive measures aimed at the general population as well as to strengthen hospital infrastructure and training of human resources. In the present study, this estimation was accurate, as observed from the real data of the beginning of the outbreak in Mexico City up to April 6, 2020. (REV INVEST CLIN. 2020;72(3):138-43)
\end{abstract}

Key words: Outbreak. Coronavirus. SARS-CoV-2. COVID-19.

Note: This manuscript has undergone an exceptional internal review process to expedite its dissemination, dwelling upon urgent information of special interest on COVID-19.

*Corresponding author:

Gustavo Cruz-Pacheco

E-mail: cruz@mym.iimas.unam.mx
Received for publication: 20-03-2020

Approved for publication: 13-04-2020

DOI: $10.24875 /$ RIC. 20000113

0034-8376 / (c) 2020 Revista de Investigación Clínica. Published by Permanyer. This is an open access article under the CC BY-NC-ND license (http://creativecommons.org/licenses/by-nc-nd/4.0/). 


\section{INTRODUCTION}

On December 31, 2019, China reported to the country's office of the World Health Organization (WHO) an outbreak of cases of pneumonia of unknown etiology. From December 31, 2020, to January 3, 2020, 44 cases of pneumonia of unknown origin in Wuhan city, Hubei Province, were reported to the WHO. On January 7 , the Chinese authorities announced that these cases were caused by a new coronavirus ( $\mathrm{nCoV}$ 2019), now called SARS-CoV- $2^{1}$. Phylogenetic analyses were immediately performed showing that the new coronavirus is related to the SARS coronavirus which emerged in Guangdong, China, in 2002 and with the MERS-CoV that emerged in Saudi Arabia in 20132,3. On January 30 , the new coronavirus was declared the sixth Public Health Emergency of International Concern according to the guidelines of the International Health Regulations ${ }^{3}$. Until March 3, the WHO reported 90,869 cases confirmed globally and 3112 deaths, from which 80,304 confirmed cases and 2946 deaths were reported by China, while 10,565 confirmed cases and 166 deaths were notified in 72 countries $^{4}$. According to the experience of the influenza pandemic due to influenza virus A (H1N1) in Mexico in 2009, it is cardinal to estimate the arrival time of the outbreak and spreading of SARS-CoV-2 in our country, to strengthen the activities according to the Health Emergency Preparedness and Response Plan for the current SARS-CoV-2 epidemic that is evolving, and from which new information is generated each day. For this reason, new methods and techniques have been developed to qualitatively understand this type of phenomenon.

\section{METHODS}

In 1927, doctors Kermack and McKendrick developed a mathematical model to study the Spanish Influenza pandemic of $1921^{5}$. This model, with some modifications, was used for forecasts and analyses of the 2009 influenza outbreak in Mexico City ${ }^{6}$. This same model, supplemented with classical diffusion (which in the context of an epidemic is managed as a dispersion of the infection), has been quite useful in studies of the spread of some infections, for example, rabies $^{7}$. In this work, we developed a new method to analyze the dissemination of a novel virus, such as SARS-CoV-2, between different cities by airline routes for the purpose of estimating the arrival of the pathogen from one city to another. Our method uses the Kermack and McKendrick model to simulate the way the outbreak grows and evolves when it arrives in the different cities, and we also use classical diffusion on a graph to model the way the infection travels between cities by the airline routes ${ }^{8}$. We used this deterministic model with classical diffusion and not a more general stochastic one because the latter models usually contain a much greater number of parameters, which are very difficult to measure for a new infection. Classical diffusion can be interpreted as a Brownian motion model, so it includes the main characteristics of a symmetric random process. The purpose of using a simple model for our analysis is to have the least number of parameters, but with enough precision to be able to give an estimate of the date of arrival of the outbreak, for instance, to Mexico City. By this date, it is well established that infection by this type of coronavirus is transmitted from person to person through droplets expelled by an infected individual when coughing or sneezing; it is also transmitted through contact with surfaces or objects contaminated with the virus and then touching the mouth, eyes, or nose' ${ }^{9}$. There is evidence that once the infectious outbreak has started, there is some homogeneity in its development in the affected city for these influenza-like infections. For these reasons, the Kermack and McKendrick model is applicable in the cities that we use as nodes of the graph, in which the edges represent the flights between one city and another.

The basic reproductive number was estimated using the data reported for Hubei and Guangdong ${ }^{10}$. An adjustment was made to the data at the beginning of the outbreak to estimate the force of infection $\lambda$, which is related to the basic reproductive number as follows: $R_{0}=1+\lambda / \beta$. The setting of $\lambda$ for Hubei and for Guangdong gives $\beta=0.3$. One over $\beta$ is a measure of the average time an infected individual is infectious. Its value from reference ${ }^{11}$ was adjusted to $\beta=0.2$ for a better fitting of the model to Wuhan's data. These estimates give an $R_{0}=2.5$, which is in very good agreement with the estimate of Wun ${ }^{11}$ of $R_{0}=2.53$ and is well into the interval of the estimations reported by read ${ }^{12}$. The diffusion coefficient $D$ was estimated by adjusting with numerical simulations. The solutions of our model to match the outbreak started in Singapore around February 15, 2020, when they 
Figure 1 . Cities considered as nodes in this analysis, as well as the corresponding graph.
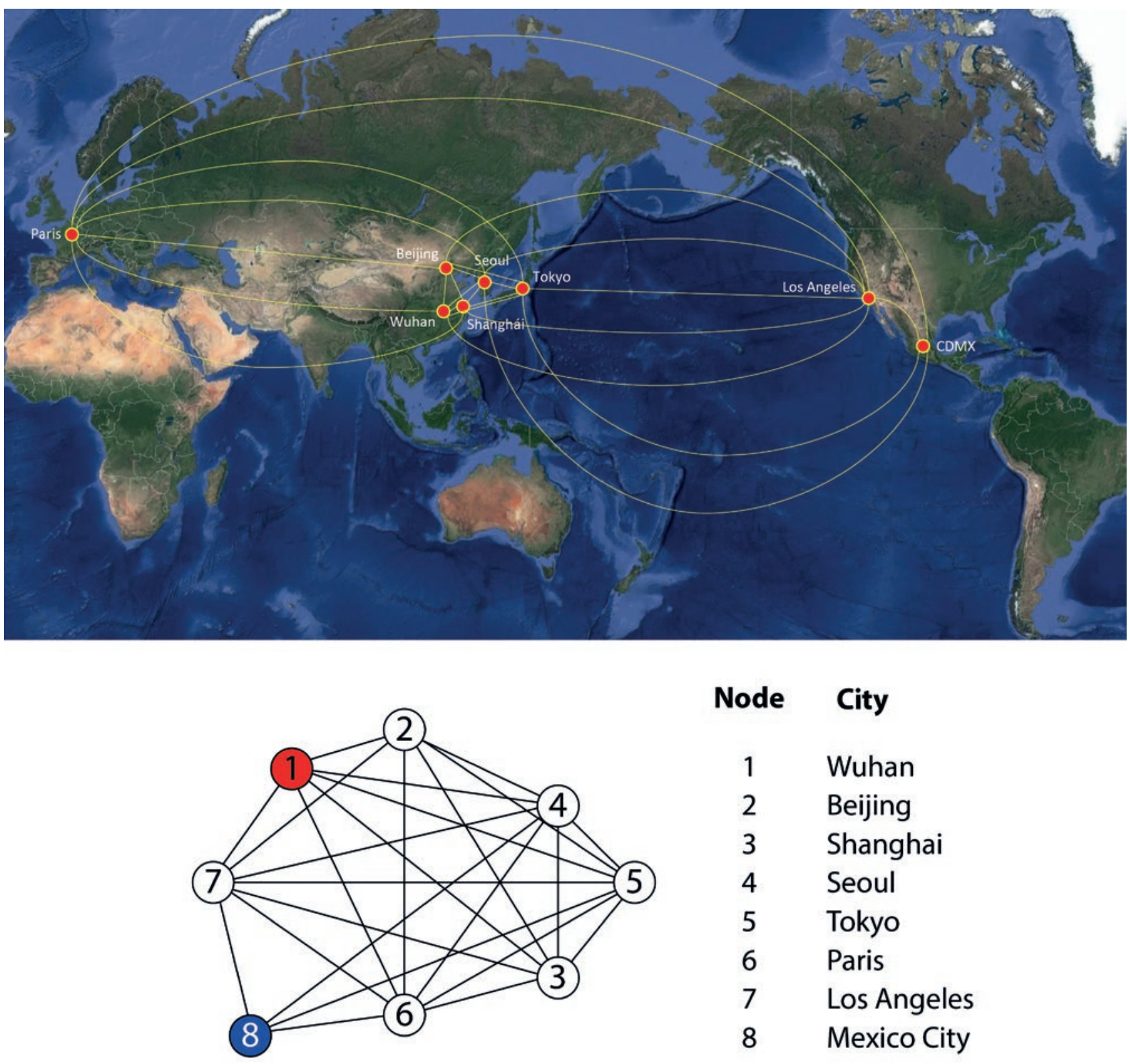

Node City

$\begin{array}{ll}1 & \text { Wuhan } \\ 2 & \text { Beijing } \\ 3 & \text { Shanghai } \\ 4 & \text { Seoul } \\ 5 & \text { Tokyo } \\ 6 & \text { Paris } \\ 7 & \text { Los Angeles } \\ 8 & \text { Mexico City }\end{array}$

registered 75 infected cases. This is an inverse method of adjusting coefficients to a differential equation; the best fit was with a diffusion coefficient $D=10^{-6}$. In Figure 1, the nodes of the graph represent cities that were chosen because their airports function as important distribution points to other airports. In the model, some of these nodes also function as a representation of other airports in the region. For example, the Paris node represents the main international airports of that European region: Paris,
Amsterdam, and Frankfurt. Using this graph and the estimated epidemiological parameters, the model was solved numerically to study in several scenarios the time of arrival of the infection in Mexico City.

\section{RESULTS}

Using the parameters estimated, the model was solved numerically, using the routine Runge-Kutta 9 
methods with the software package Wolfram Mathematica 12, to study in several scenarios the arrival of the outbreak in Mexico City. All our simulations have initial conditions in which only node 1 corresponding to the city of Wuhan has been infected, and the other cities are not yet infected or represent a very small number with respect to their populations. In the city of Wuhan, an infected number of 20 is assumed ${ }^{12}$ and January 10, 2020, was taken as the initial date for this analysis and as the starting point of the numerical simulations. The result is shown in Figure 2, where the vertical axis is the proportion of infected persons among the whole population of the city in question. The peak of the first outbreak in red corresponding to Wuhan is reached around February 24, 2020. The curves corresponding to the simulations of nodes 2-7 are identical, so they are one above the other and only the violet curve of node 7 is seen. This is because the corresponding graph is all interconnected (they form what is called a complete graph); if one excludes node 8 , the remaining graph does not distinguish one node from the others. This numerical simulation also indicates that the maximum of the first curve reaches 0.23 , which means that, at the maximum point of the graph, $23 \%$ of the urban area of Wuhan was infected. Data from Johns Hopkins University showed that the number of reported cases was peaking at approximately 63,000 . The urban area of Wuhan comprises around 20 million inhabitants (this is the population served by Wuhan airport); therefore, $23 \%$ correspond to $4,600,000$, which indicates that for each case reported, there are about 73 individuals infected in the city. In this scenario, the infectious outbreak of node 8 (peak in green), corresponding to Mexico City, begins its growth between $\mathrm{t}=70$ and $t=80$, which gives an estimate of the arrival of the outbreak in Mexico between March 20 and March 30 (blue vertical line). This information was made available to the Mexican Ministry of Health on February 24, 2020.

To simulate the isolation measures imposed on $\mathrm{Wu}$ han since the end of January, numerical simulations were performed lowering the value of $R_{0}$ from 2.5 to 2.1 first and then to 1.9; this is shown in Figure 2 by the black dashed curve for $\mathrm{R}_{0}=2.1$ and by the black curve for $R_{0}=1.9$. As it can be seen, this does not affect the height and width of the rest of the peaks and only delays the arrival of the rest of the nodes, including node 8 corresponding to Mexico City, by around 4 days. This shows that not so small changes in $R_{0}$ do not substantially alter the result of the arrival of the outbreak at node 8 . Simulations with $R_{0}=3$, when adjusted to match the outbreak in Wuhan, only anticipated the outbreak in Mexico City by 4 days. We performed other numerical simulations changing the $R_{0}$ in other nodes of the graph around $10 \%$ of its value; this changed the outbreaks at these nodes, but it did not change significantly the date of the outbreak arrival in Mexico City. Changes on the graph structure cutting some edges, meaning canceling some airline routes, did not modify importantly the date of arrival of the outbreak to the last node. Even cutting all the edges connecting to node 8 except one, only delayed the arrival of the outbreak in Mexico City around 6 days. Reducing the coefficient $D$ had a larger impact on the delay of the outbreak: reducing $D$ by one order of magnitude, from $10^{-06}$ to $10^{-07}$, delayed the arrival of the outbreak to node 8 around 2 weeks.

Figure 2 also shows an important observation: the time, at which the first node reaches the maximum moves forward as the $\mathrm{R}_{0}$ decreases, indicating that the isolation measures carried out in Wuhan only decreased the $R_{0}$ by 5 -tenths. This is because, according to the data presented by Johns Hopkins University, the report of cases begins to stabilize approximately on February 28; therefore, the maximum is expected to be reached close to this date.

\section{DISCUSSION}

This model estimates the arrival of the SARS-CoV-2 infectious outbreak in Mexico between March 20 and March 30. This estimation allows for a time window to implement and strengthen preventive measures aimed at the general population, as well as to strengthen hospital infrastructure and training of human resources. However, this period should be considered as an estimate of the maximum time of arrival of the outbreak in Mexico City, since the infection can arrive by other routes, such as sea or land through any of our borders.

This model shows that the isolation measures that can be implemented in the cities where the outbreak first arrives are very important for local control, but 
Figure 2. The curves in colors show a numerical simulation of the time evolution of an infectious outbreak that begins at node 1 , Wuhan - red curve. After peaking at node 1, it spreads to nodes 2-7 - violet curve. Finally, it arrives to node 8 , Mexico City - green curve. The curves in black show a simulation in which the $R_{0}$ has been changed as follows: $R_{0}=2.1$ (black in dashed line) and 1.9 (black). In both cases, the corresponding peaks at node 8 only slightly lagging behind.

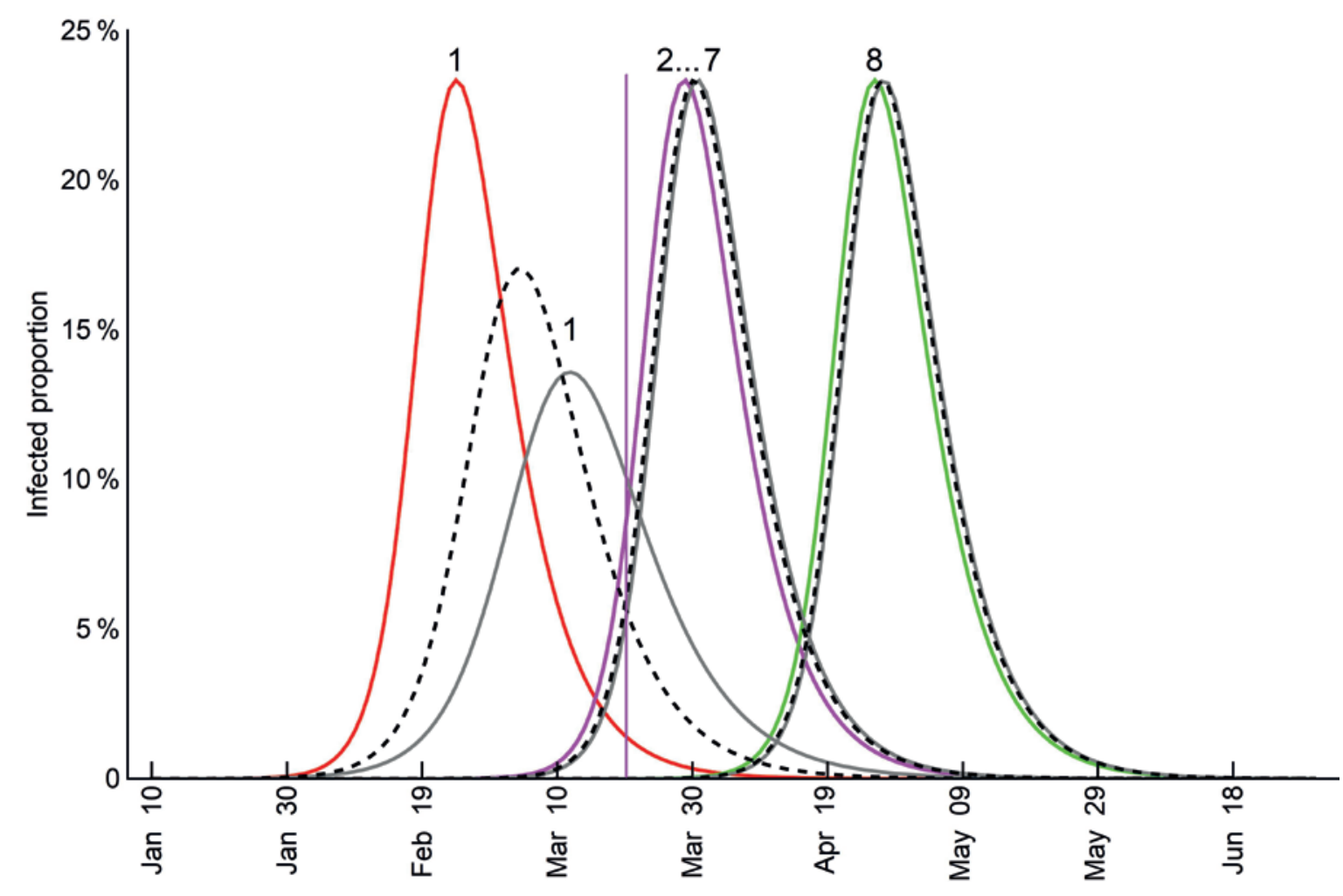

do not affect significantly the time of arrival in other cities. The coefficient that best controls the spread of infection for the rest of the cities, when this dispersion is by air travel, is the parameter $\mathrm{D}$. Therefore, surveillance at airports should be strengthened, with special emphasis on those connecting Mexico, directly or indirectly, with Asian countries. Finally, this model shows that all of these measures can only delay the arrival of SARS-CoV-2. However, if it could be delayed long enough, it would be very important to have as much time as possible to establish the appropriate prevention and control measures allowing for better management of the outbreak.

With reports of infected individuals registered by the Mexican Ministry of Health in the urban area of Mexico City up to April 6, clearly, the outbreak has begun in this region. The number of infected persons grows monotonically and at an accelerated rate, matching well an exponential fitting since around March 27, 2020. This is the first outbreak of COVID-19 in Mexico.

\section{REFERENCES}

1. World Health Organization. Novel Coronavirus (2019-nCoV). Situation Report. https://www.who.int/docs/default-source/ coronaviruse/situation-reports/20200121-sitrep1-2019-ncov.pdf?sfvrsn=20a99c10_4. [Last accessed on 2020 Mar 06].

2. Gorbalenya AE, Baker SC, Baric RS, de Groot RJ, Drosten C, Gulyaeva AA, et al. Severe Acute Respiratory Syndrome-related Coronavirus: the Species and its Viruses a Statement of the Coronavirus Study Group. bioRxiv; 2020. Available from: https://www.biorxiv.org/content/10.1101/2020.02.07.9378 62v1. [Last accessed on 2020 Feb 11].

3. Lai CC, Shih TP, Ko WC, Tang HJ, Hsueh PR. Severe acute respiratory syndrome coronavirus 2 (SARS-CoV-2) and corona virus disease-2019 (COVID-19): the epidemic and the challenges. Int J Antimicrob Agents. 2020;17:105924

4. World Health Organization. Novel Coronavirus (2019-nCoV). Situation Report 61. Available from: https://www.who.int/ docs/default-source/coronaviruse/situation-reports/ 
20200303-sitrep-43-covid-19.pdf?sfvrsn=76e425ed_2. [Last accessed on 2020 Mar 20].

5. Kermack WO, McKendrick AG. Contributions to the mathematical theory of epidemics. Proc R Soc London Ser A. 1927; 115:700-21.

6. Cruz-Pacheco G, Duran L, Esteva L, Minzoni AA, López-Cervantes $M$, Panayotaros $P$, et al. Modelling of the influenza A(H1N1) outbreak in Mexico city, April-May 2009, with control sanitary measures. Euro Surveill. 2009;14:19254.

7. Murray JD. Mathematical Biology I. An Introduction. 3rd ed. Berlin: Springer; 1989.

8. Caputo JG, Cruz-Pacheco G, Panayotaros P. Bistable reactiondiffusion on a network. J Phys A Math Theor. 2015;48:075102.

9. Centers for Disease Control and Prevention. Coronavirus Disease 2019 (COVID-19). Available from: https://www.cdc.gov/ coronavirus/2019-ncov/about/transmission.html. [Last accessed on 2020 Mar 06].

10. Coronavirus COVID-19 Global Cases by the Center for Systems Science and Engineering (CSSE) at Johns Hopkins University (JHU). Available from: https://www.coronavirus.jhu.edu/map. html. [Last accessed on 2020 Mar 06].

11. Wu JTW, Leung K, Leung GM. Nowcasting and forecasting the potential domestic and international spread of the 2019-nCoV outbreak originating in Wuhan, China: a modelling study. Lancet. 2020;395:689-97.

12. Read JM, Bridgen JR, Cummings DA, Ho A, Jewell CP. Novel Coronavirus 2019-nCoV: early Estimation of Epidemiological Parameters and Epidemic Predictions Version 2 Updated 27 Jan 2020 medRxiv, available from: https://www.medrxiv.org/content/10.1 101/2020.01.23.20018549v2. [Last accessed on 2020 Mar 06]. 